\title{
Local Density-based Hierarchical Clustering for Overlapping Distribution using Minimum Spanning Tree
}

\author{
S. John Peter \\ Department of Computer Science and Research Center \\ St. Xavier's College, Palayamkottai \\ Tamil Nadu, India.
}

\begin{abstract}
In this paper, we propose a clustering algorithm to find clusters of different sizes, shapes and densities. Density and Hierarchical based approaches are adopted in the algorithm using Minimum Spanning Tree, resulting in a new algorithm Local Density-based Hierarchical Clustering Algorithm for overlapping data distribution using Minimum Spanning Tree (LDHCODMST). The algorithm is divided into two stages. In the first stage, local density is estimated at each data point. In the second stage, hierarchical approach is used by merging clusters according to the computed cluster distance based on overlap in distribution of data points. The proposed algorithm improves the effectiveness of clustering result in which data are distributed in different shapes and different density, and that it can get a better clustering efficiency.
\end{abstract}

\section{Keywords}

Euclidean minimum spanning tree, Clustering, Core cluster, Merge similarity

\section{INTRODUCTION}

One of the best known problems in the field of data mining is clustering. The problem of clustering is to partition a data set into groups (clusters) in such a way that the data elements within a cluster are more similar to each other than data elements in different clusters [7]. Clustering is the subject of active research in several fields such as statistics, pattern recognition, machine learning, and data mining. A wide variety of clustering algorithms have been proposed for different applications [1].

Given a connected, undirected graph $G=(V, E)$, where $V$ is the set of nodes, $E$ is the set of edges between pairs of nodes, and a weight $w(u, v)$ specifying weight of the edge $(u, v)$ for each edge $(u, v) \in E$. A spanning tree is an acyclic subgraph of a graph $G$, which contains all vertices from $G$. The Minimum Spanning Tree (MST) of a weighted graph is minimum weight spanning tree of that graph. Several well established MST algorithms exist to solve minimum spanning tree problem [16, 21]. The cost of constructing a minimum spanning tree is $O(m \log n)$, where $m$ is the number of edges in the graph and $n$ is the number of vertices. More efficient algorithm for constructing MSTs have also been extensively researched $[6,5,16]$. These algorithms promise close to linear time complexity under different assumptions. A Euclidean minimum spanning tree (EMST) is a spanning tree of a set of $n$ points in a metric space $\left(\mathbf{E}^{\mathbf{n}}\right)$, where the length of an edge is the Euclidean distance between a pair of points in the point set.

Clustering algorithms using minimal spanning tree takes the advantage of MST. The MST ignores many possible connections between the data patterns, so the cost of clustering can be decreased. The MST based clustering algorithm is known to be capable of detecting clusters with various shapes and size [26]. Unlike traditional clustering algorithms, the MST clustering algorithm does not assume a spherical shapes structure of the underlying data. The EMST clustering algorithm $[20,21]$ uses the Euclidean minimum spanning tree of a graph to produce the structure of point clusters in the $n$-dimensional Euclidean space. Clusters are detected to achieve some measures of optimality, such as minimum intra-cluster distance or maximum inter-cluster distance [2]. The EMST algorithm has been widely used in practice.

Density-based clustering algorithms are designed to discover arbitrary-shaped clusters. Clusters are considered as dense regions of objects in the data space that are separated by regions of low density. DBSCAN [4] is a typical densitybased algorithm. The core point in DBSCAN is that for a fixed radius, the neighborhood of each object in a cluster has to contain at least minimum number of other objects.

The density-based clustering algorithm can also be described as a search for the connected components in a graph where the vertices correspond to the core points in the data set, and there exits an (undirected) edge between two vertices (core points) if the distance between then is less than $\varepsilon$ (ie each of them is in the neighborhood of the other point). The connected components of this graph correspond to the core points of eachcluster.

An important objective of hierarchical cluster analysis is to provide picture of data that can easily be interpreted. A picture of a hierarchical clustering is much easier for a human being to comprehend than is a list of abstract symbols. A dendrogram is a special type of tree structure that provides a convenient way to represent hierarchical clustering. A dendrogram consists of layers of nodes, each representing a cluster.

Hierarchical clustering is a sequence of partitions in which each partition is nested into the next in sequence. An Agglomerative algorithm for hierarchical clustering starts with disjoint clustering, which places each of the $n$ objects in an individual cluster [1]. The hierarchical clustering algorithm being employed dictates how the proximity matrix or proximity graph should be interpreted to merge two or more of these trivial clusters, thus nesting the trivial clusters into second partition. The process is repeated to form a sequence of nested clustering in which the number of clusters decreases as a sequence progress until single cluster containing all $n$ objects, called the conjoint clustering, remains[1].

In this paper, we propose a new Local Density-based Hierarchical Clustering for Overlapping Distribution Algorithm using Minimum Spanning Tree (LDHCODMST) which fuses the particular strength of both the DENCLUE algorithm and Hierarchical methods using Minimum Spanning Tree resulting in performance enhancements. Here we want to avoid experimental methods and advocate the idea of need-specific as opposed to case-specific because users always know the needs of their applications. We believe it is a 
good idea to allow users to define their desired similarity within a cluster and allow them to have some flexibility to adjust the similarity if the adjustment is needed. Our Algorithm produces clusters of $n$-dimensional points with a naturally approximate intra-cluster distance.

This paper is structured as follows: In section 2 we review some of the existing works on density-based clustering algorithms and hierarchical-based clustering. In Section 3 we propose LDHCODMST algorithm which produces high quality clusters. Finally in conclusion we summarize the strength of our methods and possible improvements.

\section{RELATED WORK.}

DBSCAN [4] operationalizes density propagation according to the principle "accessibility from a core point". Each points whose $\varepsilon$-neighborhood contains more points than MinPts is called a core point. Each point which lies in an $\varepsilon^{-}$ neighborhood of a core points $p$ adopts the same cluster label as $p$. The DBSCAN-algorithm propagates this relation through the set $D$. The algorithm terminates if each points is either assigned to a certain cluster or classified as noise.

Neighborhood-based Clustering (NBC) algorithm [27] proposed by Zhou S. G. et al is a good data clustering algorithm and can discover clusters of arbitrary shape and different densities using neighboring relationship among data points. To apply NBC to segment an image fast and efficiently Grayscale k-neighborhood based Density Factor (GNDF) [14] is introduced, which characterizes the local density of a gray's neighborhood in a relative sense.

DENCLUE $[10,11]$ adopts density-based clustering approach that models the overall density of a set of points as the sum of influence functions associated with each point. The resulting overall density function will have local peaks, ie., local density maxima, and these peaks can be used to define clusters in a natural way. But DENCLUE can be more computationally expensive than other density based clustering technique and it is one of the limitations of DENCLUE. YuChen Song et al [25] proposed a approach called Clustering algorithm incorporating Density and Direction, which characterizes the local density of points to find clusters of different sizes, shapes and densities

The clustering algorithm proposed by S.C. Johnson [13] uses proximity matrix as input data. The algorithm is an agglomerative scheme that erases rows and columns in the proximity matrix as old clusters are merged into new ones. The algorithm is simplified by assuming no ties in the proximity matrix. Graph based algorithm was proposed by Hubert [12] using single link and complete link methods. He used threshold graph for formation of hierarchical clustering. An algorithm for single-link hierarchical clustering begins with the minimum spanning tree (MST) for $\mathrm{G}(\infty)$, which is a proximity graph containing $n(n-1) / 2$ edge was proposed by Gower and Ross [8]. Later Hansen and DeLattre [9] proposed another hierarchical algorithm from graph coloring.

Zhan [26] proposed Minimum Spanning Tree (MST) based clustering algorithm. The MST based clustering algorithm has been widely used in practice. $\mathrm{Xu}$ (Ying), Olman and $\mathrm{Xu}$ (Dong) [23] use MST as multidimensional gene expression data. They point out that MST- based clustering algorithm does not assume that data points are grouped around centers or separated by regular geometric curve. Thus the shape of the cluster boundary has little impact on the performance of the algorithm. They described three objective functions and the corresponding cluster algorithm for computing $k$-partition of spanning tree for predefined $k>0$. The algorithm simply removes $k-1$ longest edges so that the weight of the subtrees is minimized. The second objective function is defined to minimize the total distance between the center and each data point in the cluster. The algorithm removes first $k-1$ edges from the tree, which creates a $k$-partitions.

Chowdbury and Murthy [3] proposed density oriented MSTbased clustering technique assumes that the boundary between any two clusters must belong to a valley regions(regions where the density of the data points is the lowest compared to those of the neighboring regions) and the inconsistency measure is based on the finding such valley regions.

Laszlo and Mukherjee [17] present an MST-based clustering algorithm that puts a constraint on the minimum cluster size rather than on the number of cluster. This algorithm was developed for the microaggregation problem, where the number of clusters in the data set can be figured out by the constraints of the problem itself. Vathy-Fogarassy et al [22] suggest three new cutting criteria for the MST-based clustering. Their coal is to decrease the number of heuristically defined parameters of existing algorithms so as to decrease the influence the user on the clustering results.

Grygorash et al [19] proposed two MST-based clustering algorithms, called Hierarchical Euclidean -distance based MST clustering algorithm (HEMST) and Maximum Standard Deviation Reduction clustering algorithm (MSDR), respectively. The HEMST requiring the number of clusters is given as an input. The MSDR does not require any input information about the number of cluster, is actually a recursive two-partition optimization problem.

Recently, Wang et al [24] proposed a new approach called Divide and Conquer method to facilitate efficient MST-based clustering using Reverse Delete algorithm.

\section{OUR CLUSTERING ALGORITHM}

Through this MST representation, we can convert a multidimensional clustering problem to a tree partitioning problem, i.e. finding particular set of tree edges and then cutting them. Representing a set of multi-dimensional data points as simple tree structure will clearly lose some of the inter data relationship. However many clustering algorithm proved that no essential information is lost for the purpose of clustering. This is achieved through rigorous proof that each cluster corresponds to one subtree, which does not overlap the representing subtree of any other cluster. Clustering problem is equivalent to a problem of identifying these subtrees through solving a tree partitioning problem. The inherent cluster structure of a point set in a metric space is closely related to how objects or concepts are embedded in the point set. In practice, the approximate number of embedded objects can sometimes be acquired with the help of domain experts. Other times this information is hidden and unavailable to the clustering algorithm.

\subsection{LDHCODMST Clustering}

\section{Algorithm}

LDHCODMST is an incremental algorithm. It uses the nearby data points to increase the initial clusters. If a data point meets any principle of any core cluster, the point can be 
clustered to a certain core cluster. For every single clustering event, LDHCODMST can start from any object (point) in the cluster. This does not affect the clustering result. It is important to note that only if an object having maximum density distribution value is called as density attractor. That point is considered as a core cluster center. Only a core cluster center can cluster its nearby points.

Here we describe the key concepts which are required for the first stage of the LDHCODMST algorithm.

Neighborhood Radius: for any object $x$ and distance $R$ in space, a circular region with center $x$ and radius $R$ is defined as neighborhood of objects $x$, marked as $V_{R}(x)=\{\mathrm{x} \mid 0<\mathrm{d}(\mathrm{x}$, $\left.\left.\mathrm{x}_{\mathrm{j}}\right) \leq \mathrm{R}\right\}$. The radius of the defined neighborhood can be calculated as follows

$$
R=\operatorname{mean}(D) / n^{\operatorname{coef} R}
$$

where mean $(D)$ is the mean distance among all objects and coefR is the coefficient of neighborhood radius adjustment

Object Neighborhood: For any object $x$ and distance $R$ in space, a circular region with center $x$ and radius $R$ is called neighborhood of object $\mathrm{x}$, defined as $V_{R}(x)=\left\{x \mid 0<d\left(x, x_{j}\right) \leq R\right\}$, in which $d\left(x, x_{j}\right)$ is the distance between objects $x$ and $x_{j}$.

Point Density Estimation: Given space $\Omega \in \mathrm{Fd}$ consists of a data set of $\mathrm{n}$ objects $\mathrm{D}=\left\{\mathrm{x}_{1}, \mathrm{x}_{2}, \ldots \ldots \ldots \ldots \mathrm{x}_{\mathrm{n}}\right\}$ in which density of $x_{i}$, density $P\left(x_{i}\right)$, is the value of the influence function of the object in space.

$$
P\left(x_{i}\right)=\sum_{j=1}^{n} e^{-d\left(x_{i}-x_{j}\right)^{2}} / R^{2}
$$

where the Gaussian influence function

$$
-d\left(x_{i}-x_{j}\right)^{2} / R^{2}
$$

$f_{\text {guass }}\left(x_{i}, x_{j}\right)=e^{\text {indicates the density influence }}$

of each data object to the density of object $x_{i}$, and $x_{j}$ are the points that belong to the neighborhood of the radius $R$ of $x_{i}$, $V_{R}$, and $d$ is the Euclidean distance [25].

In the algorithm proposed here, we will differentiate between two concepts: core cluster and cluster. We will refer to core clusters to the sets that are obtained after applying the first stage of the algorithm, and we will refer to the clusters to the groups of core clusters that will be grouped into clusters in a further stage.

\subsection{Merge-Similarity for MST-Hierarchical Clustering}

We define a Minimum Spanning Tree based merge-similarity measure to incorporate hierarchical approach in the second stage of the algorithm that takes into account two possible facts, when subtrees are overlapped or completely separated.

Let us define the following merge-similarity function $\mathrm{ms}$ between two subtrees $T_{i}$ and $T_{j}$,

$m s\left(T_{i}, T_{j}\right)=d o\left(T_{i}, T_{j}\right)\left(1+d s\left(T_{i}, T_{j}\right)\right)$
Where $d o\left(T_{i}, T_{j}\right)$ is a measure of overlapping between subtrees (core clusters) $T_{i}$ and $T_{j}$ and $d s\left(T_{i}, T_{j}\right)$ is a measure of separability between those subtrees.

The separability measure based on MST can be defined as

$$
\begin{aligned}
d s\left(T_{i}, T_{j}\right)= & \min \left\{d s c\left(T_{m}, T_{n}\right)\right\}, \\
& \forall T_{m}, T_{n} / T_{m} \in T_{i} \text { and } T_{n} \in T_{j}
\end{aligned}
$$

Where $T_{m}, T_{n}$ are two subtrees (core clusters), one from each subtree, and let us define the distance between two subtree as

$$
\begin{aligned}
\operatorname{dsc}\left(T_{m}, T_{n}\right) & =\min \left\{\operatorname{dist}\left(x_{m}, x_{n}\right)\right\}, \\
& \forall x_{m}, x_{n} / x_{m} \in T_{m} \text { and } x_{n} \in T_{n}
\end{aligned}
$$

That is, the distance or merge-similarity measure of separability between two subtrees (core clusters) is the shortest distance between any pair of points in the Minimum Spanning Tree, one point from each subtree (core cluster). Therefore for overlapped subtrees, $d s=0$.

On the other hand, about the subtree overlapping measure in equation (3), $d o\left(T_{i}, T_{j}\right)$, let us suppose that each subtree corresponds to one mode. A non parametric measure of the degree of overlapping of such modes can be defined referring to the density value of the border point $x_{b}$ between both modes.

Therefore, let us define the overlapping degree of the two modes $d o\left(T_{m}, T_{n}\right)$, as the relative difference between the density of the modes centers, $x_{m}$ and $x_{n}$, with respect to the density at the border $x_{b}$ between both modes. We can express this relative measure as

$$
d o\left(T_{m}, T_{n}\right)=P_{c}-P_{b} / P_{c}
$$

Given a subtree (core cluster) $T_{m}$, the centre of the subtree $x_{m}$ is defined as the point whose density is maximal within the subtree. Let $x_{m}$ and $x_{n}$ be the centers of $T_{m}$ and $T_{n}$ respectively. Therefore, $P_{c}$ in equation (5) is defined as the minimum density of the subtrees centres $x_{m}$ and $x_{n}$, that is $P_{c}=\min \left(p\left(x_{m}\right), p\left(\left(x_{n}\right)\right)\right.$. Note that the merge-similarity measure of overlapping in equation (5) is normalized in the range $[0$, $1]$.

In equation (5), $P_{b}$ is the density at the midpoint of the border between both subtrees, which is defined as the midpoint between the nearest points $x b_{m}$ and $x b_{n}$, one from each subtree, $C_{m}$ and $C_{n}$. Finally the measure of the degree of the overlapping between two subtrees $d o\left(T_{i}, T_{j}\right)$, is defined as

$$
\begin{aligned}
d o\left(T_{i}, T_{j}\right) & =\min \left\{\operatorname{doc}\left(T_{m}, T_{n}\right)\right\}, \\
& \forall T_{m}, T_{n} / T_{m} \in T_{i} \text { and } T_{n} \in T_{j}
\end{aligned}
$$

In a few words, the merge-similarity measure defined in (3) is aimed at considering that subtrees are more similar, when their probability distributions are either nearer in the feature space, measured by means of the separabilty measure $d s($ ), or when their probability distributions are more overlapped. When the probability distributions are overlapped $(d s=0)$, the measure of similarity becomes the overlapping degree of the probability density term $d o()$, which is a heuristic local 
estimate of the mixed probability distributions at the border between subtrees.

The algorithm first read data set from data base and convert into EMST. The weight of the edge in the tree is Euclidean distance between the two end points. Then it finds maximum density of points to be the cluster centers. Then it examine the remaining objects (points) by checking the distance between each object and cluster center is less than or equal to neighborhood radius $R$ and if it is does, the object belong to the subtree otherwise it does not. After the object is clustered, the object does not participate in the next clustering process and it is removed from the data set (EMST). After all the core clusters have been completed, remaining data objects will be examined to determine noise clusters in the data set.

\section{Algorithm: LDHCODMST ( )}

Input : $S$ the point set, coefR, density noise threshold Output : k clusters

Let $i$ be the number of core clusters

1. Construct a minimum Spanning Tree EMST from data set $S$

2. Compute the densities of each data objects (Points) in EMST using (2)

3. Assign each data point of the EMST to a single core cluster

4. $i=1$;

5. Repeat

6. Seek maximum density of the data point $x$

7. Compute its neighborhood radius $\mathrm{R}, V_{R}(x)$ and make the point $\mathrm{x}$ as center $\mathrm{c}_{i}$

8. Assign the objects in the EMST which are with in the radius $R$ (inside the circle) to the cluster center $\mathrm{c}_{\mathrm{i}}$ of the cluster $C_{i}$ (core cluster) and at the same time delete the clustered objects from the EMST and keep them separately

9. $i=i+1$

10. Until the data points (objects) in EMST is empty

11. Mark all the core clusters with density less than the density noise threshold as noise clusters. The rest of the core clusters are the resulting $i$ core clusters.

12. Initially, assign each one of the $i$ core clusters from the first stage to a single cluster. Therefore, there are initially $i$ clusters with one core cluster.

13. Repeat

14. Compute the merge similarity distance between each pair of core clusters using equation (3)

15. Merge the two core clusters for that the distance is minimum

16. Until $k$ clusters are obtained

22. Return $k$ clusters

The LDHCODMST algorithm works in two stages. We use figure 1 to illustrate the first stage of the algorithm which is used to create subtrees or core clusters. The first stage reads data set from database and converts into Minimum Spanning Tree EMST (line 1). Density of each object (point) is computed to identify core clusters (line 2). Next we find maximum density value of the point for identifying current core cluster center (line 6). Then points or objects which are with in the radius $R$ are assigned to core cluster center and removed from EMST and kept them separately (line 8). Lines 6-9 in the algorithm are repeated until points (objects) in the EMST become empty. Here we assume that there are 7 subtrees or core clusters (only three are shown in figure 1) are created in the first stage of the LDHCODMST algorithm. Finally noise clusters are detected based on the noise threshold value (line 11).

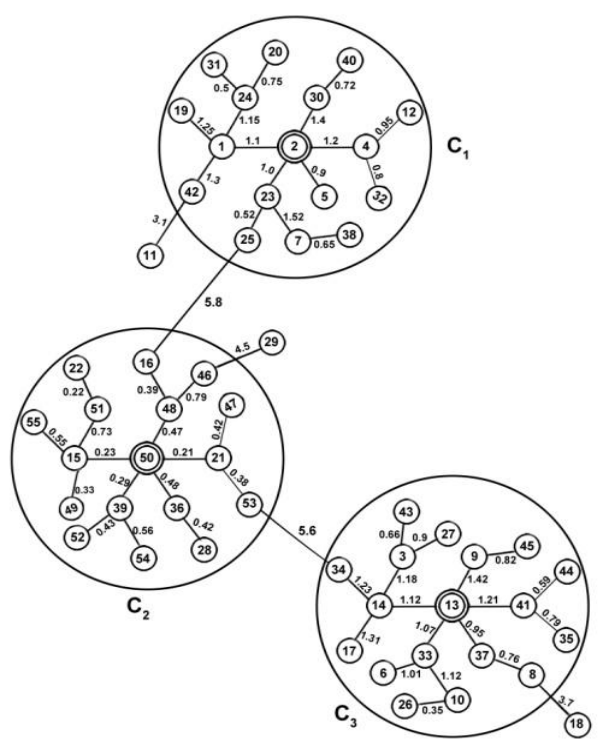

Figure 1. EMST, Three core clusters

The second stage of the LDHCODMST convert the core clusters into clusters (Dendrogram) is shown in figure 2 . Initially, assign each one of the $i$ core clusters from the first stage to a single cluster. Therefore, there are initially $i$ clusters with one core cluster (line 12). The distance between each pair of core clusters using equation (3) is computed at line 14 . Core clusters are merged according to the measure of distance computed using the equation (3) is at line 15. The lines 14 and 15 in the second stage of the algorithm are repeated until the $k$ number of clusters are obtained. The dendrogram for $k$ number of cluster as objects is generated. The objects within the clusters are compact. The clusters are well separated, shown in Figure 2.

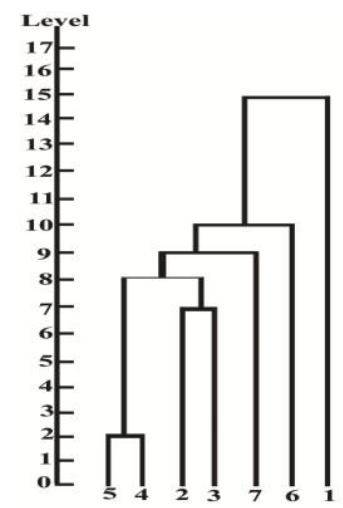

Figure 2. Dendrogram using 7 core clusters with centers and noise clusters 


\section{CONCLUSION}

Our LDHCODMST clustering algorithm is a hierarchical algorithm based on local density information. The way the density of the probability distribution is estimated, and the use of this information in the introduced dissimilarity measure between clusters, provides to the algorithm a mechanisms to deal with overlapping distributions and presence of noise in the data set. The first stage of the algorithm gradually finds core clusters with center for each of them. The second stage produces clusters based on hierarchical approach. These clusters ensure guaranteed intra-cluster similarity. Our algorithm does not require the users to select and try various parameters combinations in order to get the desired output. All of these look nice from theoretical point of view. However from practical point of view, there is still some room for improvement for running time of the clustering algorithm. This could perhaps be accomplished by using some appropriate data structure. In the future we will explore and test our proposed clustering algorithm in various domains. We will further study the rich properties of EMST-based clustering methods in solving different clustering problems.

\section{REFERENCES}

[1] Anil K. Jain, Richard C. Dubes "Algorithm for Clustering Data", Michigan State University, Prentice Hall, Englewood Cliffs, New Jersey 07632.1988.

[2] T. Asano, B. Bhattacharya, M.Keil and F.Yao. "Clustering Algorithms based on minimum and maximum spanning trees". In Proceedings of the $4^{\text {th }}$ Annual Symposium on Computational Geometry, Pages 252-257, 1988.

[3] N. Chowdhury and C.A. Murthy, “ Minimum Spanning Tree -Based Clustering Techniques: Relationship with Bayes Classifier", Pattern Recognition, Vol. 30, no 11, pp 1919-1929, 1997.

[4] M. Ester, H.-P. Kriegel, J. Sander, and X. Xu. "A DensityBased Algorithm for Discovering Clusters in Large Spatial Databases with Noise". In Proceedings of the 2nd International Conference on Knowledge Discovery and Data Mining (KDD96), 1996.

[5] M. Fredman and D. Willard. "Trans-dichotomous algorithms for minimum spanning trees and shortest paths". In Proceedings of the $31^{\text {st }}$ Annual IEEE Symposium on Foundations of Computer Science, pages 719-725, 1990.

[6] H.Gabow, T.Spencer and R.Rarjan. "Efficient algorithms for finding minimum spanning trees in undirected and directed graphs", Combinatorica, 6(2):109-122, 1986.

[7] S. Guha, R. Rastogi, and K. Shim. "CURE an efficient clustering algorithm for large databases". In Proceeding of the 1998 ACM SIGMOD Int. Conf. on Management of Data , pp 73-84, Seattle, Washington, 1998.

[8] J.C. Gower and G.J.S. Ross "Minimum Spanning trees and single-linkage cluster analysis" Applied Statistics 18, 5464, 1969.

[9] P. Hansen and M. Delattre, "Complete-link cluster analysis by graph coloring" Journal of the American Statistical Association 73, 397-403, 1978.

[10] A. Hinneburg and D.A. Keim, "An Efficient Approach to Clustering in Large Multimedia Databases with Noise," In Proc. Of th 4th Intl. Conf. on Knowledge Discovery and Data Mining, pp. 58-65. 1998.
[11] A. Hinneburg and D.A. Keim, "A General Approach to Clustering in Large Databases with Noise," Knowledge and Information Systems (KAIS), vol. 5, no. 4, pp. 387 $415,2003$.

[12] Hubert L. J "Min and max hierarchical clustering using asymmetric similarity measures" Psychometrika 38, 63$72,1973$.

[13] S. C. Johnson, "Hierarchical clustering schemes" Psychometrika 32, 241-254, 1967.

[14] Jundi Ding, SongCan Chen, RuNing Ma and Bo Wang, "A Fast Directed Tree Based Neighborhood Clustering Algorithm for Image Segmantation", Neural Information Processing, Lecture Notes in Computer Science, Vol 4233,pp 369-378, 2006.

[15] D. Karger, P. Klein and R. Tarjan, "A randomized lineartime algorithm to find minimum spanning trees". Journal of the ACM, 42(2):321-328, 1995.

[16] J. Kruskal, "On the shortest spanning subtree and the travelling salesman problem", In Proceedings of the American Mathematical Society, pp 48-50, 1956.

[17] M. Laszlo and S. Mukherjee, "Minimum Spanning Tree Partitioning Algorithm for Micro aggregation", IEEE Trans, Knowledge and Data Eng, Vol. 17, no 7, pp $902-$ 911, July 2005.

[18] J. Nesetril, E.Milkova and H.Nesetrilova. Otakar boruvka on "Minimum spanning tree problem": Translation of both the 1926 papers, comments, history. DMATH: Discrete Mathematics, 233, 2001

[19] Oleksandr Grygorash, Yan Zhou, Zach Jorgensen. "Minimum spanning Tree Based Clustering Algorithms". Proceedings of the $18^{\text {th }}$ IEEE International conference on tools with Artificial Intelligence (ICTAI'06) 2006.

[20] F. Preparata and M.Shamos. "Computational Geometry": An Introduction. Springer-Verlag, Newyr, NY ,USA, 1985.

[21] R. Prim. "Shortest connection networks and some generalization”. Bell systems Technical Journal,36:13891401, 1957.

[22] A. Vathy-Fogarassy, A. Kiss, and J. Abonyi , "Hybrid Minimal Spanning Tree and Mixture of Gaussians Based Clustering Algorithms", Proc. IEEE Intl Conf. Tools with Artificial Intelligence, pp 73-81, 2006.

[23] Y.Xu, V.Olman and D.Xu. "Minimum spanning trees for gene expression data clustering". Genome Informatics, 12:24-33, 2001.

[24] Xiaochun Wang, Xiali Wang and Mitchell Wilkes, "A Divide-and-Conquer Approach for Minimum Spanning Tree Based Clustering", IEEE Trans, Knowledge and Data Eng, Vol. 21 no 7 , pp 945-958, July 2009.

[25] Yu-Chen Song, J.O'Grady,G.M.P.O'Hare, Wei Wang,“A Clustering Algorithm incorporating Density and Direction", IEEE Computer Society, CIMCA 2008.

[26] C. Zahn. "Graph-theoretical methods for detecting and describing gestalt clusters". IEEE Transactions on Computers, C-20:68-86, 1971.

[27] Zhou, S., Zhao, J.: "A Neighborhood-Based Clustering Algorithm”. PAKD 2005, LNAI 3518 361-371 , 1982. 